\title{
The potentially beneficial role of an aortic arch anatomical variant
}

\author{
Gabor Erdoes, MD · Stefanos Demertzis, MD • \\ Reto Basciani, MD • Zsolt Szuecs-Farkas, MD, PhD • \\ Thierry Carrel, MD • Balthasar Eberle, MD
}

Received: 6 June 2010/Accepted: 7 July 2010/Published online: 20 July 2010

(C) Canadian Anesthesiologists' Society 2010

\section{To the Editor,}

In recent years, surgical and anesthetic techniques for brain protection have improved markedly in thoracic aortic surgery. Nevertheless, central nervous system injury is the complication that remains most feared, as it contributes significantly to overall morbidity and mortality. ${ }^{1}$ We discuss a case of acute type-A aortic dissection requiring a complex repair of the aorta wherein an unexpected anatomic variant of the innominate artery allowed immediate institution of bilateral antegrade cerebral perfusion (ACP).

A previously healthy 57 -yr-old man was admitted to a district hospital after he had suffered a syncopal episode on a ski run. He was found unconscious in the snow after an unknown time period had elapsed. After recovering consciousness, he complained to the rescue team of severe chest pain. Transthoracic echocardiography revealed a large pericardial effusion and moderate aortic regurgitation. This medical condition along with other clinical signs (syncope, pulse asymmetry, and chest pain) led to a tentative diagnosis of type-A aortic dissection. With hemodynamics deteriorating and acute respiratory failure imminent, the computed tomography scan in the district facility was cancelled. While the patient was on high-dose vasoactive support, his trachea was intubated and his lungs were ventilated prior to urgent transfer to our centre. In the operating room, a transesophageal echocardiography examination confirmed the diagnosis of type-A aortic dissection. Extracorporeal circulation was established with the

G. Erdoes, MD $(\bowtie) \cdot S$. Demertzis, MD $\cdot$ R. Basciani, MD ·

Z. Szuecs-Farkas, MD, PhD - T. Carrel, MD - B. Eberle, MD

Bern University Hospital, Bern, Switzerland

e-mail: Gabor.Erdoes@insel.ch arterial inflow cannula inserted in the right axillary artery and a two-stage venous drainage cannula inserted in the right atrium. On reaching the targeted nasopharyngeal temperature $\left(20^{\circ} \mathrm{C}\right)$, hypothermic circulatory arrest was instituted. By cross-clamping the innominate artery at its origin, the surgeon sought to establish a continuous unilateral right axillary ACP via the right-sided carotid and vertebral artery. The exploration of the aortic arch revealed a circumferential dissection with multiple intimal tears extending beyond the ostium of the innominate artery. However, only one additional arch vessel ostium could be identified. Further preparation of the distal aortic arch showed that the second arch ostium did not, as previously assumed, represent the left common carotid artery, but
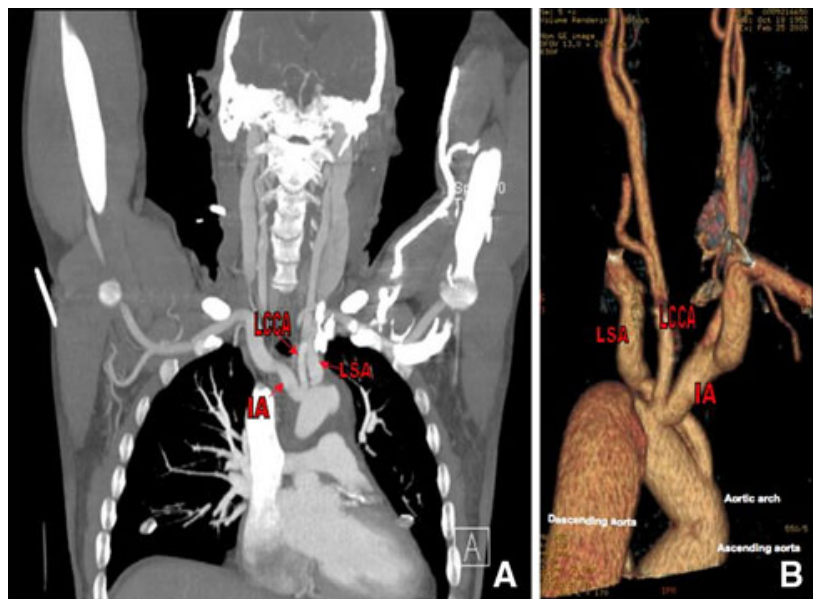

Fig. 1 A) Coronal reconstruction of the computed tomography angiography of the chest (frontal view) and B) surface rendering of the aortic arch (posteroanterior view) in the postoperative course. The common origin of the innominate artery (IA) with the left common carotid artery (LCCA) is apparent. The left subclavian artery (LSA) was re-implanted in the aortic arch 


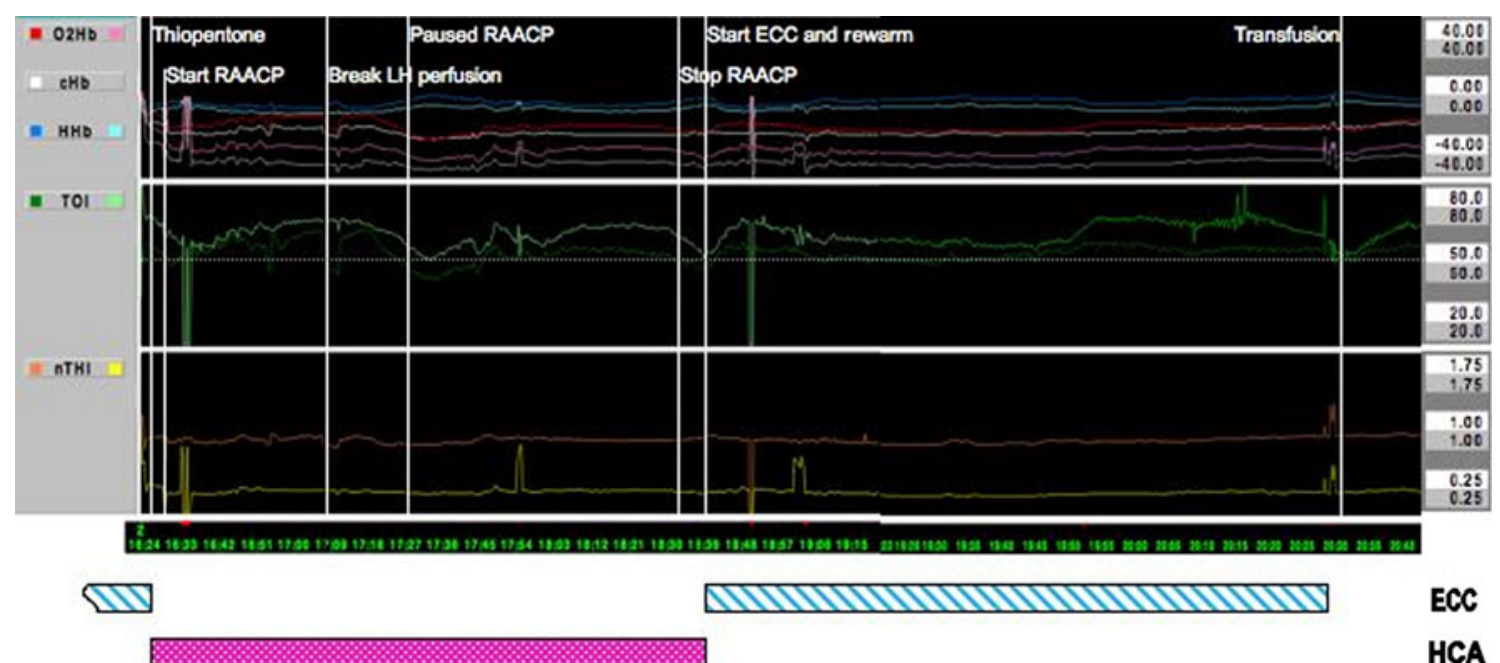

Fig. 2 Near-infrared oximetry record during hypothermic circulatory arrest and extracorporeal circulation in the phase of re-warming / weaning off. The TOI curves of both hemispheres are predominantly $>45 \%$, corresponding stable frontoparietal cerebral oxygenation over the entire operation. The detected decreases of TOI are marked in the figure: at 17:06 pm, reduced perfusion of the left hemisphere due to brief unblocking of the perfusion catheter placed in

rather the left subclavian artery. Additional preparation of the innominate artery identified the origin of the left common carotid artery directly from the innominate ostium (Fig. 1). This anatomical variant of the brachiocephalic vessel had rendered the presumably unilateral ACP as being bilateral from the beginning of hypothermic circulatory arrest.

Throughout hypothermic circulatory arrest and ACP, continuous bilateral near-infrared oximetry monitoring (NIRS) demonstrated stable frontoparietal cortical oxygen saturation $>45 \%$ (Fig. 2). The processed electroencephalogram Bispectral Index (BIS ${ }^{\mathrm{TM}}$ ) that became isoelectric following cooling recovered to normal levels during re-warming. Separation from cardiopulmonary bypass was uneventful. The patient was eventually weaned from the respirator and his trachea extubated on postoperative day five. He was discharged from the hospital after two weeks without focal neurological deficits.

With widespread adoption of advanced thoracic aortic surgery, anatomic variants of the aortic arch may interfere more often with introducing ACP. In urgent cases, thorough preoperative image-based evaluation of supra-aortic vessel anatomy may not be possible. Therefore, identification and cannulation of arch vessels for ACP may be error-prone in the presence of anomalous anatomy and may require adaptations of the cerebral protection methods. However, a common origin of the innominate and left common carotid arteries, "bovine arch", 2,3 as presented in this report, was a fortunate constellation allowing early, effective, and uninterrupted bilateral ACP. the left subclavian artery; at 17:27 pm, the RAACP was paused temporarily due to bleeding near the innominate artery. $\mathrm{O}_{2} \mathrm{Hb}=$ oxyhemoglobin; $\mathrm{cHb}=$ total hemoglobin; $\mathrm{HHb}=$ deoxyhemoglobin; TOI $=$ tissue oxygen index; $\mathrm{nTHI}=$ normalized tissue hemoglobin index; RAACP = right axillary antegrade cerebral perfusion; $\mathrm{LH}=$ left hemisphere; $\mathrm{ECC}=$ extracorporeal circulation; $\mathrm{HCA}=$ hypothermic circulatory arrest

The optimal method for cerebral protection during hypothermic circulatory arrest is still a matter of discussion. However, in cases of prolonged hypothermic circulatory arrest ( $>40 \mathrm{~min}$ ) as reported by our group, ${ }^{4}$ bilateral ACP provides the best method of cerebral protection, resulting in stable bi-hemispheric oxygenation values in NIRS monitoring ${ }^{5}$ and decreased risk of stroke. ${ }^{6}$ We believe that early institution of bi-hemispheric ACP may have contributed to the favourable neurological outcome of this patient. In contrast, other anomalies of the supra-aortic vessels, if not recognized, can preclude sufficient cerebral protection. For this reason, we recommend the routine use of more than one cerebral monitoring technique, i.e., multimodal neuromonitoring, ${ }^{7}$ especially in emergency aortic surgery without the possibility of preoperative imaging of supra-aortic vessels.

Competing interests None declared.

\section{References}

1. Krahenbuhl ES, Immer FF, Stalder M, Englberger L, Eckstein FS, Carrel TP. Temporary neurological dysfunction after surgery of the thoracic aorta: a predictor of poor outcome and impaired quality of life. Eur J Cardiothor Surg 2008; 33: 1025-9.

2. Jakanani GC, Adair W. Frequency of variations in aortic arch anatomy depicted on multidetector CT. Clin Radiol 2010; 65: 481-7.

3. Kaul P, Javangula K, Ganti S, et al. Continuous selective bilateral antegrade cerebral perfusion through anomalous innominate artery for repair of root, ascending aorta and arch aneurysm-challenges, 
vagaries and opportunities of bovine arch variant anatomy and review of literature. Perfusion 2009; 24: 121-33.

4. Krahenbuhl ES, Clement M, Reineke D, et al. Antegrade cerebral protection in thoracic aortic surgery: lessons from the past decade. Eur J Cardiothorac Surg 2010; 38: 46-51.

5. Harrer $M$, Waldenberger FR, Weiss $G$, et al. Aortic arch surgery using bilateral antegrade selective cerebral perfusion in combination with near-infrared spectroscopy. Eur J Cardiothorac Surg 2010; DOI:10.1016/j.ejcts.2010.03.016.
6. Olsson $C$, Thelin $S$. Antegrade cerebral perfusion with a simplified technique: unilateral versus bilateral perfusion. Ann Thorac Surg 2006; 81: 868-74.

7. Erdos G, Tzanova I, Schirmer U, Ender J. Neuromonitoring and neuroprotection in cardiac anaesthesia. Nationwide survey conducted by the Cardiac Anaesthesia Working Group of the German Society of Anaesthesiology and Intensive Care Medicine (German). Anaesthesist 2009; 58: 247-58. 